\title{
Efecto de la fertilización orgánica y densidad para la producción de granadilla Passiflora ligularis Juss eco tipo Colombiana en la Comunidad Campesina San Miguel de Tabaconas
}

\section{Effect of organic fertilization and density for the production of passion fruit Passiflora ligularis Juss eco type Colombiana in the San Miguel de Tabaconas Peasant Community}

\author{
Miguel Galecio ${ }^{1}$ (D)* , Teresa Peña ${ }^{2}$ (D) Ricardo Peña ${ }^{3}$ iD y Bartolomé Rojas $^{4}$ (D)
}

\begin{abstract}
RESUMEN
La granadilla Passiflora ligularis Juss es una especie de importancia económica del género Passiflora, por su fruto nutritivo y comercialización en los mercados nacionales e internacionales. De ahí la necesidad de asumir mayor conocimiento de tecnologías agronómicas para un aprovechamiento sostenible. El experimento se realizó en La Bermeja - Tabaconas, San Ignacio Región Cajamarca - Perú. Se evaluó el efecto de fertilización orgánica dosis y densidades de siembra: dosis baja: $(100,60,80)$, media $(150,100,110)$, alta $(205,184,180) \mathrm{N}, \mathrm{P}_{2} \mathrm{O}_{5}, \mathrm{~K}_{2} \mathrm{O} \mathrm{kg}$. ha- 1 , con densidades de siembra $(277,500$ y 833 plantas.ha-1), en el cultivo de granadilla eco tipo Colombiana. El diseño experimental que se utilizó fue de bloques completos al azar con tres repeticiones. La mejor respuesta de fertilización se obtuvo con los tratamientos T3 (100-60-80 kg.ha-1 de NPK, densidad de siembra alta de 833 plantas.ha1) y T2 (100-60-80 kg.ha-1 de NPK, densidad de siembra alta de 500 plantas.ha-1) que permitieron obtener el mayor número de frutos.planta-1, número de frutos .ha-1 y rendimiento de fruta en kg.ha-1, con valores de 513, 18481 y 2640.14 respectivamente para el T3 y 321, 17852 y 2550.29 respectivamente para el T2, los cuales fueron estadísticamente similares entre sí, pero, sólo el tratamiento T3 superó estadísticamente al resto de tratamientos en el periodo de cosecha octubre - enero.
\end{abstract}

Palabras clave: Guano de isla, sulfato de potasio, densidad, dosis, rendimiento, granadilla.

\begin{abstract}
The passion fruit Passiflora ligularis Juss is a species of economic importance of the genus Passiflora, due to its nutritious fruit and commercialization in national and international markets. Hence the need to assume greater knowledge of agronomic technologies for sustainable use. The experiment was carried out in La Bermeja - Tabaconas, San Ignacio Cajamarca Region - Peru. The effect of organic fertilization doses and planting densities was evaluated: low dose: $(100,60,80)$, medium $(150,100,110)$, high $(205$, $184,180) \mathrm{N}, \mathrm{P}_{2} \mathrm{O}_{5}, \mathrm{~K}_{2} \mathrm{O}$ kg.ha- 1, with planting densities $(277,500$ and 833 plants.ha-1), in the cultivation of Colombian-type eco-passion fruit. The experimental design that was used was a randomized complete block with three repetitions. The best fertilization response was obtained with the treatments T3 (10060-80 kg.ha-1 of NPK, high sowing density of 833 plants.ha-1) and T2 (100-60-80 kg.ha-1 of NPK, high planting density of 500 plants.ha-1) that allowed to obtain the highest number of fruits.plant-1, number of fruits .ha-1 and fruit yield in kg.ha-1, with values of 513, 18481 and 2640.14 respectively for T3 and 321,17852 and 2550.29 respectively for T2, which were statistically similar to each other, but only the T3 treatment statistically surpassed the rest of the treatments in the October-January harvest period.
\end{abstract}

Keywords: Island guano, potassium sulfate, density, dose, yield, passion fruit.

DOI: https://doi.org/10.37787/pakamuros-unj.v8i3.136

Recibido: 25/08/2020. Aceptado: 04/10/2020

* Autor para correspondencia

Universidad Nacional de Piura, Piura, Perú, Email: miguelgalecio9@ hotmail.com

Consultora Privada, Piura, Perú, Email: tere_1019@hotmail.com

Universidad Nacional de Piura, Piura, Perú:Email:ripecastillo@gmail.com

Investigador Privado, Jaén, Email: investigacioncenfrocafe@gmail.com.pe 


\section{INTRODUCCIÓN}

La granadilla es la segunda especie de importancia económica del género Passiflora, por su fruto comestible y comercialización en los mercados internacionales. Ocampo et al., (2015). Está catalogada como exótica, lo que le confiere un gran potencial de exportación Agronet., (2015). Esta es originaria de la región de los Andes de Perú a Colombia, y en la actualidad está distribuida desde Argentina hasta México, en altitudes que oscilan entre 1400 y 2200 m.s.n.m. aunque se produce en Costa de Marfil, Sur de África, Kenia y Australia. Es común encontrarla creciendo de forma silvestre en la región comprendida entre Argentina y México de zonas tropicales (Cerdas y Castro, 2003; Ocampo et al., 2007). El año 2017 en el Perú la cosecha fue de 6043 ha, donde las regiones que obtuvieron mayor producción fueron: Pasco, Junín, Huánuco y Cajamarca con 23000, 14000, 4400 y 3400 miles de toneladas respectivamente (MINAGRI 2018). Para el cultivo de granadilla solo el 5,30 \% de los productores realiza análisis de suelos; $24,58 \%$ a veces realiza la fertilización mineral, mientras que el $16.76 \%$ nunca emplea y solo el $13.97 \%$ siempre emplea y un alto porcentaje no riega las plantaciones de granadilla (Camasa, 2019). Además en la actualidad es muy común observar una intensa agricultura del tipo convencional, en diferentes zonas del valle de Oxapampa, especialmente en la producción del cultivo de la granadilla, donde los agricultores son protagonistas dependientes del uso indiscriminado de pesticidas, lo cual está generando todo un desequilibrio en el ecosistema y agroecosistema causando contaminación en los frutos, del suelo y en las aguas de escorrentía debido una concentración masiva de metales pesados muy tóxicos (Buendia, 2018).

De otro lado, Fischer y Álvarez., (2008) y Aular et al., (2014) indican que un buen manejo del cultivo aumenta el potencial de la variedad. Siendo la nutrición de las plantas uno de los factores clave para el desarrollo de la calidad del fruto y debido a su efecto en el crecimiento, influye mucho en la producción y calidad del fruto. Agregan Bejarano, e Ibarra, (2018), que los múltiples factores que influyen en el proceso de producción para el incremento de rendimientos entre ellos, la fertilización. Incorporando lo relacionado con los factores climáticos y los sistemas agrarios de fácil y rápida adaptación del ecotipo "Colombiana" a las características edafoclimáticas de los ecosistemas, lo que coadyuvó con la mejora del rendimiento local del cultivo, la intensidad del manejo del cultivo en los sistemas agrarios y el cambio de uso de la tierra, contribuyeron con el incremento en el rendimiento de granadilla. Igualmente, Rivera et al., (2002), incide que la exigencia nutricional es la cantidad de nutriente que una planta exige extraer del suelo para cumplir su período productivo y generar un rendimiento esperado. Para los productores de granadilla del distrito de Oxapampa, la aplicación de materia orgánica es eventual y entre las 
principales necesidades de innovación, destaca la fertilización y la totalidad de los productores adoptó el sistema de conducción de emparrado, el eco tipo colombiano (Beyer, 2018).

Para la densidad de plantas en el cultivo de granadilla, se considera que la distancia de siembra, debe variar con la fertilidad de los suelos, la topografía y el manejo del cultivo (Bernal y Tamayo, 1999). En el caso de Borja, (2019) encontró que el método de siembra de 5 x $5 \mathrm{~m}$. es el distanciamiento mejor adaptado para la producción de granadilla en emparrado y que el manejo con el sistema emparrado facilitó realizar las diferentes labores agronómicas entre ellas la fertilización, obteniéndose incrementar los rendimientos. Así otro estudio demuestra que el cultivo de granadilla, es rentable, equitativo, resiliente, con debilidades en la parte ambiental, que requiere nuevas innovaciones tecnológicas para lograr la sostenibilidad del cultivo (Romero, 2019).

Por ello, la investigación tuvo el objetivo de evaluar el efecto de la dosis de fertilización y densidad de siembra de granadilla eco tipo "Colombiana" orgánica en la Comunidad Campesina san Miguel de Tabaconas - Distrito de Tabaconas, tratando de encontrar dosis óptimas sin afectar los recursos suelo, agua y aire, con el uso de insumos para agricultura orgánica.

\section{MATERIALES Y MÉTODOS}

\section{Área de estudio}

La investigación se realizó en el Distrito de Tabaconas que se encuentra ubicado en la parte Suroeste de la Provincia de San Ignacio entre los $05^{\circ} 18^{\prime} 53^{\prime \prime}$ de latitud Sur y $79^{\circ} 16^{\prime} 55^{\prime \prime}$ de longitud Oeste, en la Región Cajamarca. La parcela demostrativa La Bermeja ubicada a (1599 m.s.n.m.), Latitud Sur (5²4' 44 “); longitud Oeste (79 13' 59.4“), se ejecutó en el periodo comprendido entre junio 2017 - julio 2019. En el distrito, posee un clima que va del frío a templado-cálido, alcanzando temperaturas en el día hasta $\operatorname{los} 27^{\circ} \mathrm{C}$ y por las noches hasta $1^{\circ} \mathrm{C}$, con épocas marcadas de invierno (enero - abril) o verano (julio noviembre), con precipitaciones pluviales promedio anual 700 - $1170 \mathrm{~mm}$ y humedad relativa promedio anual de 65\% Aguilar, (2011). Respecto a la zona de vida corresponde a bosque húmedo premontano tropical (Bh-PT) basado en la clasificación de (Holdridge, 1967).

\section{Desarrollo del cultivo}

El cultivo de granadilla Passiflora ligularis Juss se instaló en la parcela demostrativa La Bermeja en las mismas condiciones de la zona en la fase de almácigo y trasplante, labores de manejo agronómico. El almacigo se realizó en camas almacigueras con sustrato de la zona turba, ceniza y tierra agrícola luego fueron trasplantadas a bolsa almaciguera de $9 \mathrm{~cm}$. x $18 \mathrm{~cm}$, con 7 a $10 \mathrm{~cm}$. cm de altura, posteriormente 
a los 45 a 60 días fueron llevadas y trasplantadas a campo definitivo de acuerdo al diseño experimental con densidades de siembra baja (6 m. x 6 m. 277 plantas ha-1); media (5 m. x 4 m. 500 plantas. ha-1) y $4 \mathrm{~m} . \times 3 \mathrm{~m} .833$ plantas. ha-1). Las labores de deshierbo se ejecutaron con moto guadaña tipo plateo, control de plagas y enfermedades aplicando caldo sulfocálcico, control cultural y etológico. Se aplicaron cinco fertilizaciones cada 45 días. Realizando la primera cosecha a los 14 meses entre los meses de octubre a enero (Cerdas y Castro 2003).

\section{Diseño}

Se utilizó un diseño completamente al azar con tres repeticiones. Se estudiaron tres dosis de fertilización orgánica: baja 100 N, $60 \mathrm{P}_{2} \mathrm{O}_{5}, 80 \mathrm{~K}_{2} \mathrm{O}$ NPK (kg. ha-1); media 150, 100, 110 NPK (kg. ha-1); alta 205, 184, 180 NPK (kg. ha-1). y densidades de siembra: baja (6 x 6 m. = 277 plantas. ha-1); media (4 x 5 m. $=500$ plantas. ha-1) y alta $(3 \times 4 \mathrm{~m} .=833$ plantas. ha-1), lo que originó un total de 9 tratamientos, tres repeticiones, tres bloques (Tabla 1). EL área experimental fue homogénea para la 27 sub parcelas, el número de granadillas cosechadas es equivalente a 5 plantas. Se utilizaron un campo de cultivo eco tipo Colombiana; usando fuentes de guano de Islas $10 \% \mathrm{~N} ; 12 \% \mathrm{P}_{2} \mathrm{O}_{5} ; 3 \% \mathrm{~K}_{2} \mathrm{O}$; Bioeurope $12.5 \% \mathrm{~N}$; Silicio Orgánico $26.62 \% \mathrm{SiO}_{2}$; Sulfato de Potasio $\mathrm{K}_{2} \mathrm{SO}_{4} 50 \% \mathrm{~K}_{2} \mathrm{O}$ y $18 \% \mathrm{~S}$.

Tabla 1 Combinaciones o tratamientos

\begin{tabular}{|c|c|c|c|c|}
\hline \multirow{2}{*}{$\begin{array}{c}\text { Trata. } \\
\text { T1 }\end{array}$} & Dosis de fertilización $\left(\mathrm{kg} . \mathrm{ha}^{-1}\right)$ & \multirow{2}{*}{$\frac{\mathbf{x}}{\text { Baja }}$} & \multicolumn{2}{|c|}{ Densidad de plantas (plantas. ha $^{-1}$ ) } \\
\hline & $100 \mathrm{~N}, 60 \mathrm{P}_{2} \mathrm{O}_{5}, 80 \mathrm{~K}_{2} \mathrm{O}$ & & $(6 \times 6 \mathrm{~m}=277)$ & Densidad baja \\
\hline $\mathrm{T} 2$ & $100 \mathrm{~N}, 60 \mathrm{P}_{2} \mathrm{O}_{5}, 80 \mathrm{~K}_{2} \mathrm{O}$ & Dosis Baja & $(4 \times 5 \mathrm{~m}=500)$ & Densidad media \\
\hline $\mathrm{T} 3$ & $100 \mathrm{~N}, 60 \mathrm{P}_{2} \mathrm{O}_{5}, 80 \mathrm{~K}_{2} \mathrm{O}$ & Dosis Baja & $(3 \times 4 \mathrm{~m}=833)$ & Densidad alta \\
\hline $\mathrm{T} 4$ & $150 \mathrm{~N}, 100 \mathrm{P}_{2} \mathrm{O}_{5}, 110 \mathrm{~K}_{2} \mathrm{O}$ & Dosis Media & $(6 \times 6 \mathrm{~m}=277)$ & Densidad baja \\
\hline $\mathrm{T} 5$ & $150 \mathrm{~N}, 100 \mathrm{P}_{2} \mathrm{O}_{5}, 110 \mathrm{~K}_{2} \mathrm{O}$ & Dosis Media & $(4 \times 5 \mathrm{~m}=500)$ & Densidad media \\
\hline T6 & $150 \mathrm{~N}, 100 \mathrm{P}_{2} \mathrm{O}_{5}, 110 \mathrm{~K}_{2} \mathrm{O}$ & Dosis Media & $(3 \times 4 \mathrm{~m}=833)$ & Densidad alta \\
\hline $\mathrm{T} 7$ & $205 \mathrm{~N}, 184 \mathrm{P}_{2} \mathrm{O}_{5}, 180 \mathrm{~K}_{2} \mathrm{O}$ & Dosis Alta & $(6 \times 6 \mathrm{~m}=277)$ & Densidad baja \\
\hline $\mathrm{T} 8$ & $205 \mathrm{~N}, 184 \mathrm{P}_{2} \mathrm{O}_{5}, 180 \mathrm{~K}_{2} \mathrm{O}$ & Dosis Alta & $(4 \times 5 \mathrm{~m}=500)$ & Densidad media \\
\hline T9 & $205 \mathrm{~N}, 184 \mathrm{P}_{2} \mathrm{O}_{5}, 180 \mathrm{~K}_{2} \mathrm{O}$ & Dosis Alta & $(3 \times 4 \mathrm{~m}=833)$ & Densidad alta \\
\hline
\end{tabular}

\section{Parámetros de rendimiento}

Los parámetros de evaluación fueron: número de granadillas, siendo un promedio de dos plantas cosechadas en cada subparcela; número de granadillas/plantas/ subparcelas, referida $180 \mathrm{~m}^{2}$, considerando el factor en estudio densidad de plantas; de manera que en dicha área número de frutos por planta, número de frutos. ha-1 y rendimiento de $\mathrm{kg}$. ha- ${ }^{-1}$ 


\section{Análisis estadístico}

Se utilizó bloques completos al azar en parcelas divididas, con el factor dosis de fertilización en parcelas y el factor densidad de plantas en subparcelas, en tres bloques.

Cabe anotar que, finalmente, en análisis de varianza se hizo en forma equivalente al diseño experimental

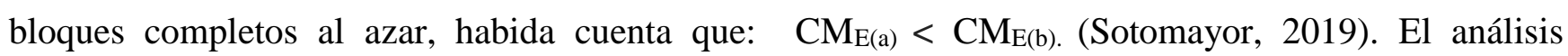
estadístico, de análisis de varianza y Duncan 0.05, se hizo con la observación de: número de granadillas /plantas/subparcelas, referida 180 metros cuadrados, con la transformación de datos: Transe (Número de granadillas / plantas / subparcelas, referido a $180 \mathrm{~m}^{2}$. Se empleó el programa IBM SPSS Statistics.

\section{RESULTADOS}

\section{Características químicas de los suelos en estudio}

En la Tabla 2, se muestran el análisis fisicoquímico del suelo con clase textural franco arcilloso. Para el cultivo de la granadilla son ideales suelos de textura franca (franco, franco arenoso); profundidad efectiva de $60 \mathrm{~cm}$, en los primeros $30 \mathrm{~cm}$. se encuentran la mayor concentración de raíces activas de la planta (50 al 60\%), autores aseveran que el $\mathrm{pH}$ del suelo, el rango óptimo esta entre 5,5 a 6,5 apropiado para el cultivo. También mencionan que es preferible cultivar con suelos que tengan un $\mathrm{pH}$ entre 6,1-7,5 (Cerdas y Castro, 2003; Rivera et al., 2002; Lim, 2012). Estos resultados coinciden con los resultados donde el pH es ligeramente ácido, con un valor de 6,33. Contenido de materia orgánica fue 0,28 \%, nitrógeno, 0,01\%; fósforo disponible $8 \mathrm{ppm}$ medio; potasio asimilable fue $133 \mathrm{ppm}$, conductividad eléctrica de 0.60 dS.m-1. La capacidad de Intercambio catiónico fue 17,85 chol (+). kg-1. Los cationes Ca++, Mg++, $\mathrm{Na}+\mathrm{y} \mathrm{K}+$, con valores de 12,$51 ; 3,15 ; 0,34$ y $0,32 \mathrm{cmol}(+) . \mathrm{kg}-1$, con $36 \%, 32 \%$ y $32 \%$ de arena, limo y arcilla respectivamente de textura franco arcillosa. Luego de la interpretación el contenido de nutriente que aporta el suelo con valores desde $39.60 \mathrm{~N}, 27,20 \mathrm{P}_{2} \mathrm{O}_{5}$ y $167,69 \mathrm{~K}_{2} \mathrm{O}$ (kg. ha-1) de contenido respectivamente.

Tabla 2. Interpretación de análisis de suelos parcela experimentales La Bermeja - Tabaconas

\begin{tabular}{lccc}
\hline \multicolumn{1}{c}{ Determinación } & Unidad & Método & Resultados \\
\hline & & & Franco \\
Clase textural & $\%$ & Bouyoucos & Arcilloso \\
Arena & $\%$ & & 36 \\
Limo & $\%$ & & 32 \\
Arcilla & & Potenciómetro & 32 \\
$\mathrm{pH}(1: 2.5)$ & & & 6,33
\end{tabular}


Materia orgánica

Nitrógeno total

Calcáreo (CaCO3)

Fósforo disponible

Potasio asimilable

Conductividad Eléctrica

Capacidad de Intercambio Catiónico

$\mathrm{Ca}$

$\mathrm{Mg}$

$\mathrm{Na}$

$\mathrm{K}$

$\begin{array}{cc}\text { Walkley y Black } & 0,28 \\ \text { Estimado a partir de mat. orgánica } & 0,01 \\ \text { Volumétrico } & 0,00 \\ \text { Olsen } & 8 \\ \text { Espectrofotometría } & 133 \\ \text { Sumatoria de bases cambiables } & 0,6 \\ \text { Acetato de amonio } & 17,85 \\ \text { Complejométrico } & 12,51 \\ \text { Complejométrico } & 3,15 \\ \text { Complejométrico } & 0,32 \\ \text { Complejométrico } & 0,34\end{array}$

0,28

,01

8

133

17,85

12,51

3,15

0,34

\section{Número de frutos de granadilla por planta}

En la Tabla 3, no fueron constatados efectos significativos, la prueba de Duncan 0,05, no detecta diferencia de los factores densidad de siembra y dosis de fertilización NPK, tanto en la interacción como en forma individual sobre el número de frutos por planta, numero de frutos por ha y rendimiento $\mathrm{kg}$. ha1. A pesar que no se apreció efecto significativo interacción de los factores individuales, el mayor número de frutos promedio por planta fueron obtenidos con dosis baja el tratamiento T3: dosis 100, 60, 80 NPK $\left(\mathrm{kg} . \mathrm{ha}^{-1}\right.$ ) para densidad (833 plantas. ha ${ }^{-1}$ ) y $\mathrm{T} 2$ densidad (500 plantas. ha ${ }^{-1}$ ) con la misma dosis, obteniendo un valor de 513 y 321 frutos. planta $^{-1}$ respectivamente. El menor valor lo obtuvo el tratamiento T1 con dosis $\left(100,60,80\right.$ NPKkg.ha $\left.{ }^{-1}\right)$ x baja densidad de 277 plantas. ha ${ }^{-1}$ con un valor de 77 frutos en promedio por planta.

\section{Rendimiento Número de frutos. ha-1}

Al observar la Tabla 3, el mejor tratamiento fue T3 con dosis: 100, 6080 NPK (kg. ha $\left.{ }^{-1}\right)$ para densidad (833 plantas. ha ${ }^{-1}$ ), con un rendimiento de 18,481 frutos ha ${ }^{-1}$ y el Tratamiento T2 dosis: 100, 60, 80 NPK $\left(\mathrm{kg}\right.$. ha ${ }^{-1}$ para densidad (500 plantas. ha ${ }^{-1}$ ) con rendimiento de 17852 frutos. ha ${ }^{-1}$ en comparación a los menores valores numéricamente similares de los tratamientos T5, T9 y T8 con valores de 15685, 15148 y 12444 (frutos. ha ${ }^{-1}$ ); continúan descendiendo los tratamientos T6 y T4 con 8481 y 7241 frutos. ha ${ }^{-1}$ y finalmente los más bajos rendimientos fueron los tratamientos T7 y T1 con valores de 4687 y 4259 frutos. $\mathrm{ha}^{-1}$ respectivamente Figura 1.

\section{Rendimientos de granadilla $\mathrm{kg} \cdot \mathrm{ha}^{-1}$}

La extracción de nutrientes depende del genotipo, la edad, estado fenológico; además de factores externos relacionados con el ambiente donde se desarrolla la planta como la temperatura, la humedad relativa, el brillo solar, la temperatura del suelo, entre otras (Sancho, 2007). Entre tanto, Guerrero, (2001) sostiene que los nutrientes extraídos en cultivo de granadilla de $160 \mathrm{~N}, 18 \mathrm{P}_{2} \mathrm{O}_{5}, 170 \mathrm{~K}_{2} 0,115 \mathrm{Ca}$ y $10 \mathrm{Mg}$. (kg. $\mathrm{ha}^{-1}$ ). Para la investigación coincide al menos con los macronutrientes en $\mathrm{N}>\mathrm{K}>\mathrm{P}>$. 
En la Tabla 3, se muestra el mayor rendimiento en granadilla obtenido fue con el T3: 100, 60, 80 NPK $\left(\mathrm{kg} \mathrm{ha}^{-1}\right)$ densidad 833 plantas. ha $\left.{ }^{-1}\right)$ con un rendimiento de 2640,14 kg. ha ${ }^{-1}$, significativo fue superior al

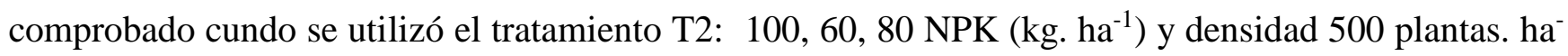
$\left.{ }^{1}\right)$ con rendimiento de $2550,29 \mathrm{~kg} \mathrm{ha}^{-1}$ similar estadísticamente, en comparación a los menores valores numéricamente similares de los tratamientos T5, T9 y T8 con valores de 2241 y $2164 \mathrm{~kg}$. ha- ${ }^{-1}$; luego continúan disminuyendo los tratamientos T6 y T4 con 1211.,7 y 1034,43 kg. ha ${ }^{-1}$ y finalmente los más bajos rendimientos fueron los tratamientos T7 y T1 con valores de 669.57 y $608 \mathrm{~kg}$. ha ${ }^{-1}$ respectivamente (Figura 2).

Tabla 3. Producción de granadilla orgánicas en la Comunidad Campesina San Miguel de Tabaconas. La Bermeja Duncan 0,05: Número de granadilla/planta/referidas a ha.

\begin{tabular}{ccccc}
\hline \multirow{2}{*}{ Tratamiento } & $\mathbf{N}^{\mathbf{0}}$ de frutos.planta- $^{\mathbf{1}}$ & $\mathbf{N}^{\mathbf{0}}$ de frutos.ha- $^{\mathbf{1}}$ & Duncan $\mathbf{0 . 0 5}$ & Rendimiento kg.ha $^{\mathbf{1}}$ \\
\hline T3 & $513 * *$ & $18,481 * *$ & $\mathrm{a}$ & $2640.14 * *$ \\
T2 & $321 * *$ & $17,852 * *$ & $\mathrm{ab}$ & $2550.29 * *$ \\
T5 & $282 \mathrm{n} \mathrm{s}$ & $15,685 \mathrm{n} \mathrm{s}$ & $\mathrm{abc}$ & $2240.71 \mathrm{n} \mathrm{s}$ \\
T9 & $273 *$ & $15,148 *$ & $\mathrm{abc}$ & $2164.00 *$ \\
T8 & $224 *$ & $12,444 *$ & abcd & $1777.71 *$ \\
T6 & $153 \mathrm{n} \mathrm{s}$ & $8,481 \mathrm{n} \mathrm{s}$ & $\mathrm{bcd}$ & $1211.57 \mathrm{n} \mathrm{s}$ \\
T4 & $130 \mathrm{n} \mathrm{s}$ & $7,241 \mathrm{n} \mathrm{s}$ & $\mathrm{cd}$ & $1034.43 \mathrm{n} \mathrm{s}$ \\
T7 & $84 \mathrm{n} \mathrm{s}$ & $4,687 \mathrm{n} \mathrm{s}$ & $\mathrm{d}$ & $669.57 \mathrm{n} \mathrm{s}$ \\
T1 & $77 \mathrm{n} \mathrm{s}$ & $4,259 \mathrm{n} \mathrm{s}$ & $\mathrm{d}$ & $608.43 \mathrm{n} \mathrm{s}$ \\
\hline
\end{tabular}

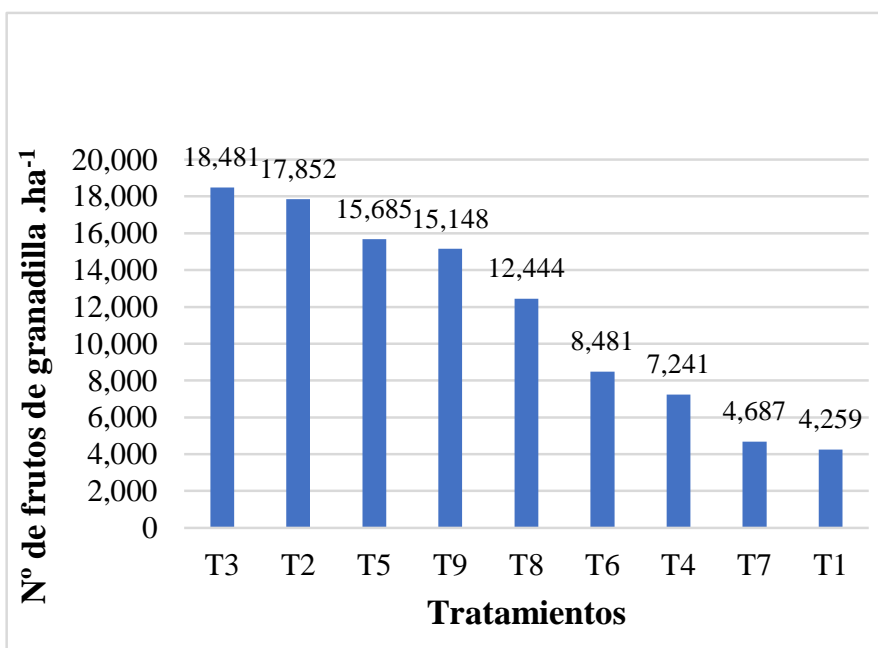

Figura 1. Rendimiento de granadilla en número de frutos ha-1

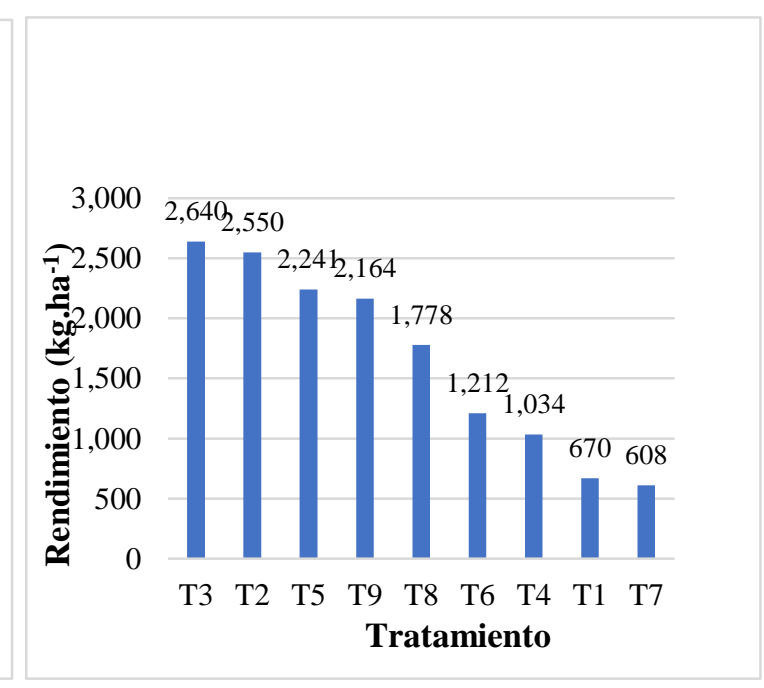

Figura 2. Rendimiento de granadilla por tratamiento. 


\section{DISCUSIÓN}

En la Tabla 3, se muestran los promedios del número de frutos, el análisis según la prueba de Duncan 0,05 indican que el factor densidad es significativo, que T3: densidad 833 plantas. ha ${ }^{-1}$, y con dosis baja de fertilización 100, 60, 80 NPK (kg. ha-1) proporcionó un valor promedio de 513 frutos. planta ${ }^{-1}$, resultando de similar respuesta estadística con el T2 con la misma dosis 100, 60, 80 NPK (kg. ha $\left.{ }^{-1}\right), \mathrm{y}$ densidad (500 plantas $\mathrm{ha}^{-1}$ ), se obtuvo valores de 321 frutos, planta ${ }^{-1}$, superiores respecto al resto de tratamientos y el testigo. Teniendo en cuenta que la investigación se realizó en un suelo franco arcilloso, investigaciones como la de Cabrera, (2006) arrojó que rendimiento aceptable a un cultivo de 6 a 8 años de edad, obtuvo una producción de 640 a 1120 frutos. planta ${ }^{-1}$. Valores muy superiores a los obtenidos en la investigación en promedio 513 y 321 frutos en promedio por planta de los mejores tratamientos (Tabla 3), debido que se obtuvo la primera cosecha. En cambio, coinciden a lo investigado por Morillo, (2017), obtuvo resultados de investigación en el cantón Pimampiro, Ecuador (2200 m.s.n.m.) el rendimiento va desde los 100 a 300 unidades por planta.

El mejor tratamiento Tabla 3, fue T3 con dosis: 100, 6080 NPK (kg. ha ${ }^{-1}$ ) para densidad (833 plantas. $\left.\mathrm{ha}^{-1}\right)$, con un rendimiento de 18,481 frutos. $\mathrm{ha}^{-1}$. Resultado que coincide sobre todo en la densidad con Álvarez et al., (2018) que los mejores rendimientos por hectárea en maracuyá amarillo se han informado cuando las densidades de siembra fueron 667 y 833 plantas. En tanto, el tratamiento T2 dosis: 100, 60, 80 NPK (kg. ha ${ }^{-1}$ para densidad (500 plantas. ha ${ }^{-1}$ ) con rendimiento de 17852 frutos. ha ${ }^{-1}$ Figura 1, concuerda con la investigación por Castro, (2001) que, con una distancia de 5 m. x 5 m. (400 plantas. ha $\left.{ }^{1}\right)$ se alcanzan altos rendimientos, mejor desarrollo de la planta y mayor longevidad del cultivo. Se tiene muchos reportes de investigación en cultivo de maracuyá como referente de las pasifloras como lo aportes por Eber, (2016) las densidades: 1600 (D1); 2666; 3200 (D3), (plantas ha-1) pudo verificar que la densidad de siembra del maracuyá amarilla más adaptada al sur de Brasil, con mayor rendimiento productivo, es de 3200 plantas. ha ${ }^{-1}$ y la calidad del fruto no fue influenciada por las densidades de siembra. Igualmente, para cultivo de granadilla con densidades de 555, 625 (plantas. ha ${ }^{-1}$ ) con sistema parral indican ser más apropiado (Tello, 2013; Morillo, 2017). Importante lo que encontró Nunes et al., (2017) investigaciones en maracuyá amarilla menciona que, a pesar de la disminución de la productividad de la primera a la segunda cosecha, el biofertilizante bovino, asociado con la fertilización de potasio aumentó el rendimiento de la cosecha del estado de Paraíba, Brasil. Además, de una población de 833 de alta densidad ha demostrado mayor número de frutos y existe potencial para trabajar. Sin embargo, un exceso de follaje favoreció la mayor presencia de enfermedades como botrytis Botrytis cinerea. Y 
menores valores de rendimiento en la investigación, que correspondió a una primera cosecha (octubre diciembre) y desarrollado en un ambiente de agricultura bajo secano.

El cultivo de la granadilla, durante las etapas reproductivas comprendidas por la formación de los botones florales, floración, crecimiento y maduración de los frutos, demanda gran cantidad de agua. Las condiciones de estrés hídrico a las que son sometidos los cultivos durante las temporadas secas pueden afectar los rendimientos en la producción al ocasionarse daños como deshidratación y caída de flores, formación de frutos pequeños o caída de ellos, golpe de sol, cuarteamiento de frutos y deficiencias nutricionales, principalmente (Fernández et al., 2014). Dentro de las pasifloras, la producción de fruta de maracuyá amarilla (Passiflora edulis Sims. F. Flavicarpa Deg.) se ve afectada por varios factores, como el clima, el suelo, las prácticas agrícolas, incluida la fertilización y el riego, que son esenciales para un alto rendimiento de los cultivos (Silva et al., 2016). Factor a tener en cuenta también lo investigado por Suárez et al., (2016) en sus resultados demostró que $P$. ligularis es una especie autofértil, pero el flujo génico y la productividad dependen de los agentes polinizadores. Estos polinizadores deben ser conservados e integrados en los planes de manejo del cultivo, debido a que proporcionan un servicio reproductivo y ecosistémico. En granadilla, la intervención de abejorros (Xylocopa sp) incrementa significativamente la formación de los frutos en un 70\% (Arias et al., 2016).

El máximo rendimiento de granadilla Tabla 3, fue de 2640,14 kg. ha-1 obtenido con una densidad de densidad 833 plantas. ha ${ }^{-1}$ y dosis de fertilización: 100, 60, 80 NPK (kg. ha-1). La misma dosis y con densidad de 500 plantas. ha ${ }^{-1}$ se logró un rendimiento de $2550,29 \mathrm{~kg}$. ha ${ }^{-1}$ superiores en relación al resto de tratamientos Figura 2. Entre tanto, los mayores rendimientos, encontró Tello, (2013), con distanciamiento entre planta de $\left(6.0 \mathrm{~m}\right.$. x $5 \mathrm{~m}$. = 333 plantas. ha $\left.{ }^{-1}\right)$ presentó los mayores valores promedios en rendimiento $(35437,1) . \mathrm{kg}$. ha ${ }^{-1} \mathrm{y}$ con la distancia entre calles de $(3.0 \mathrm{~m}$. $\mathrm{x} 2.5 \mathrm{~m}=1333$ plantas. ha ${ }^{-1}$ ) mostró un rendimiento de (31013.9) $\mathrm{kg} \mathrm{ha}^{-1}$. Además, de una población de 833 de alta densidad ha demostrado mayor. Para la investigación fueron utilizados como fuente de nitrógeno fértil orgánico $12.5 \% \mathrm{~N}$; Guano de Isla $10 \% \mathrm{~N}, 12 \% \mathrm{P}_{2} \mathrm{O}_{5}, 3 \% \mathrm{~K}_{2} \mathrm{O}$ y roca fosfórica $18.3 \% \mathrm{P}_{2} \mathrm{O}_{5}$ y Sulfato de potasio $50 \% \mathrm{~K}_{2} \mathrm{O}$, fuentes permitidas para la producción orgánica que coincide con la dosis baja de 100,60, 80 NPK kg. ha-1 con los resultados de 2640,14 kg. ha ${ }^{-1} ; 2550,29 \mathrm{~kg} \mathrm{ha}^{-1}$ y una dosis de fertilización de 100, 60, 80 NPK (kg. ha-1) para ambos casos la producción de granadilla eco tipo colombiana. Este resultado puedo estar relacionado con los factores edáficos relacionados con las propiedades físicas, químicas, biológicas y fertilidad del suelo. La combinación de estos factores define las categorías de aptitud para el cultivo en zonas aptas, medianamente aptas, marginales y no aptas 
(Miranda 2012). En el similar sentido Rajan et al., (2011) revela, que determinar la capacidad de una zona o región para producir cosechas dentro del clima genera un efecto directo sobre la fenología del cultivo, especialmente la temperatura y la precipitación. En cambio, Borja, (2019), utilizando al trasplante abono orgánico (20 kg.) más 30 g. de fosfato diamónico por hoyo), y la dosis de 68, 91, 66 NPK (kg. ha ${ }^{-1}$ ) obtuvo 7000 kg. ha ${ }^{-1}$, en granadilla eco tipo colombiana. Del mismo modo Morillo, (2017) utilizo la dosis de: 120, 80, 160 NPK $\left(\mathrm{kg} . \mathrm{ha}^{-1}\right)(1 \mathrm{~kg}$ de Nitrofoska azul, $110 \mathrm{~g}$ de súper fosfato tripe y $160 \mathrm{~g}$ de urea por planta) mas $5 \mathrm{~kg}$. planta ${ }^{-1}$ de materia orgánica, obtuvo 10473,5 kg. ha ${ }^{-1}$ independiente de poda de dos o tres ejes utilizando densidad 625 plantas. ha ${ }^{-1}$ con el eco tipo colombiana. Entre tanto Suárez y Tomalá (2012), investigaron una dosis en San Vicente La Libertad, Ecuador en cultivo de maracuyá con dosis de 150, 40, 100 NPK (kg. ha ${ }^{-1}$ ), 1250 kg. Guano de Isla; 125 kg $\mathrm{P}_{2} \mathrm{O}_{5}$ y 200 kg K $2 \mathrm{O}$ (833 g. planta ${ }^{-1} ; 133$ g. planta $^{-1}$ ), con una densidad de 666 plantas. ha ${ }^{-1}$ (5 m x 3 m.) obteniendo $8540 \mathrm{~kg}$. $\mathrm{ha}^{-}$. Resultados muy similares bajo las condiciones de Sullana Piura, obtuvieron un rendimiento de 10589,87 Kg. ha ${ }^{-1}$, con 64 frutos/planta por cosecha con madurez comercial en cultivo de maracuyá (Peña y Galecio 2019). A su vez Rivera et al., (2002) durante los primeros meses se registró 2 t. ha-1 luego los rendimientos incrementaron a un promedio en los materiales evaluados $\left(5.4 \mathrm{t}\right.$. ha $\left.{ }^{-1}\right)$. Además de los resultados de Camasa, (2019) resultados obtenidos en el Distrito Santa Teresa La Convención Cusco fue de 3.05 t. ha ${ }^{-1}$, rendimiento por debajo del promedio nacional de $5.97 \mathrm{t}^{\text {t. ha }}{ }^{-1}$, baja la competitividad de esta cadena productiva. Dosis de nutrientes que coinciden con lo investigado, con diferentes fuentes de uso para agricultura convencional. En este sentido, en futuras investigaciones, ir practicando poda de hojas y tallos, para permitir una mejor ventilación en la plantación, que facilite el control de plagas y enfermedades y un adecuado desarrollo de las plantas. Arguedas-García et al., (2017), Al agregar, cantidades de materia orgánica para complementar a los fertilizantes inorgánicos, así como aumentar la acumulación de C y $\mathrm{N}$ en el suelo. Tirol et al., (2007). A su turno, Rivera, (2016); realizó el fraccionamiento hasta 6 veces por año fraccionando el contenido de Nitrógeno, fósforo y potasio con aplicaciones de 42; 74, 110, 130; 164 y 190 g. planta $^{-1}$ para el ciclo de producción. Con $28.17 \%$ para N, P y K y micronutrientes $15.49 \%$. Varias investigaciones, destacan el rol de los nutrientes, como las investigaciones en condado de Juazeiro, Brasil, donde mayores rendimientos de maracuyá amarilla se registraron a dosis de 290 y $350 \mathrm{~kg}$. ha ${ }^{-1} \mathrm{~N}$ sin y con sustancias húmicas, respectivamente. Silva et al., (2016). Igualmente, la aplicación de fertilizante de potasio en los tratamientos de riego deficitario aumentó el rendimiento respecto al control (Bahrani y Pourreza, 2016). 
Por su parte, Criollo y García, (2009) señalaron que con el aumento de la densidad de plantación disminuye, por lo general, la biomasa por planta, aunque esta se incrementa por unidad de superficie. Todo lo contrario, Noda y Martín, (2014) mencionan que la utilización de altas densidades permite hacer un uso más intensivo de la tierra, mejor aprovechamiento del cultivo, la planta es capaz de sobrevivir en estas condiciones siempre que cuente con un suministro de nutrientes. Igualmente, Romero (2019), requiere nuevas innovaciones tecnológicas para lograr la sostenibilidad del cultivo. En ese sentido, la falta de mayor respuesta a la aplicación de macronutrientes NPK en la investigación, ya sea en forma de factor como en interacción con la densidad de plantas y de su dosis de fertilización, sobre el número de frutos por planta, número frutos. $\mathrm{ha}^{-1} \mathrm{y} \mathrm{kg}$. ha ${ }^{-1}$, se debe seguramente, a que los nutrientes sobre la zona experimental no fueron solubilizados por la presencia de pérdida de humedad del suelo para el eco tipo Colombiana, en razón de una menor eficiencia de utilización de los nutriente por la planta, debido a diversos procesos edáficos por efecto de las condiciones climáticas presentes por el periodo experimental, pueden haber sumado para lograr obtener ese resultado.

\section{CONCLUSIONES}

La mejor respuesta de fertilización se obtuvo con los tratamientos T3 (100-60-80 kg.ha-1 de NPK, densidad de siembra alta de 833 plantas.ha-1) y T2 (100-60-80 kg.ha-1 de NPK, densidad de siembra alta de 500 plantas.ha-1) que permitieron obtener el mayor número de frutos.planta-1, número de frutos .ha-1 y rendimiento de fruta en $\mathrm{kg}$.ha-1, con valores de 513, 18481 y 2640.14 respectivamente para el T3 y 321, 17852 y 2550.29 respectivamente para el T2, los cuales fueron estadísticamente similares.

Sólo el tratamiento T3, superó estadísticamente al resto de tratamientos, al cual le corresponde la dosis de fertilización baja 100, 60, 80 NPK (kg. ha-1) que equivale a 240 g de guano de Isla; 89.6 g de bioeurope (12.5 g de N) y $49.60 \mathrm{~g}$. de sulfato de potasio por/planta con cinco abonamientos.

\section{AGRADECIMIENTOS}

Al programa de Investigación e Innovación Agraria PNIA, por el co financiamiento en la ejecución del proyecto. A las Instituciones Comunidad Campesina San Miguel de Tabaconas, ejecutora; Cooperativa de Servicios Múltiples Global Café; Corporación Alto Marañón SAC; Municipalidad Distrital de Tabaconas Universidad Nacional de Piura como entidades colaboradoras. 


\section{REFERENCIAS BIBLIOGRÁFICAS}

Aguilar, J. (2011). Instalación de seiscientas hectáreas de especies Agroforestales, en la Cuenca del rio Tabaconas, micro cuencas Alisal, Manchara, Charape y Urubamba del Distrito de Tabaconas, Provincia de San Ignacio- Cajamarca-Municipalidad Distrital de Tabaconas 123 p.

Álvarez, H., J. Pionce, J. Castro, W. Viera, y A. Sotomayor. 2018. Densidades poblacionales y fertilización nitrogenada en maracuyá. Ecuador es Calidad: Rev. Cient. Ecuatoriana 5(1):1-6.

Agronet. (2015). Sistema de estadísticas agropecuarias. En: http://www.agronet.gov.co/agronetweb1/Estad\%C3\%ADsticas.aspx. .

Arias, C., Ocampo, J., y Urrea, R. (2016). Sistemas de polinización en granadilla (Passiflora ligularis Juss.) como base para estudios genéticos y de conservación. Acta Agronomica, 65(2), 197-203.

Arguedas, C. y Monge, J. (2017). Efecto de la densidad de siembra sobre el rendimiento y calidad de los frutos de dos genotipos de berenjena (Solanum melongena L.) cultivados en invernadero en Costa Rica. Revista Tecnología en Marcha, 30(4), 66-79.

Aular, J., Casares, M., y Natale, W. (2014). Nutrição mineral e qualidade do fruto do abacaxizeiro e do maracujazeiro. Rev. Bras. Frutic 36 (4), 1046-1054. Doi: 10.1590 / 0100-2945-269 / 14

Bahrani, A. y Pourreza, J. (2016). Effect of alternate furrow irrigation and potassium fertilizer on seed yield, water use efficiency and fatty acids of rapeseed. Idesia (Arica), 34(2), 35-41.

Bernal, A. y Tamayo, J. (1999). Informe de Visita a municipios productores de Granadilla del Departamento de Caldas. Rionegro. CORPOICA Regional 4. Colombia 20 p.

Bejarano, A. y Ibarra, D. (2018). Impacto de la variabilidad climática y de los sistemas agrarios en el cultivo de granadilla (Passiflora ligularis Juss) de Oxapampa, Pasco, Perú. Biotempo, 15(1), 4148.

Beyer, A. (2018). Adopción del emparrado en Passiflora ligularis (Juss.) y su contribución al desarrollo local de Oxapampa, Perú. Tesis de Magister Universidad Agraria La Molina 109 pp.

Borja, J. (2019). Experiencia en manejo técnico de la granadilla (Passiflora ligularis) en el Alto Chicama-Otuzco. Tesis de Ingeniero Universidad Privada Antenor Orrego Trujillo - Perú 64 p. Buendia, B. (2018). Evaluación de la concentración de metales pesados en frutos de (Passiflora ligularis Juss) por uso intensivo de agroquímicos Oxapampa, Pasco. Universidad Nacional del Centro del Perú - Huancayo Perú. 143 pp. 
Cabrera, C. (2006). Caracterización de las propiedades físicas y químicas del fruto de granadilla, Passiflora ligularis juss. Tesis de Ingeniero. Ibarra: Universidad Técnica del Norte - Ecuador.

Camasa, J. (2019) Factores de Competitividad en la Cadena Productiva de Granadilla (Passiflora ligularis Juss) en Santa Teresa, La Convención, Cusco. Tesis de Magister. Universidad Agraria La Molina Lima - Perú 111 pp.

Castro, J. (2001). Guía básica para el establecimiento y mantenimiento del cultivo de la granadilla (Passiflora ligularis Juss.). Bogotá, ASOHOFRUCOL. Fondo Nacional del Fomento Hortofrutícola. 75 pp.

Cerdas, M. y Castro, J. (2003). Manual práctico para la producción, cosecha y manejo poscosecha del cultivo de granadilla (Passiflora ligularis Juss). Ministerio de Agricultura y Ganadería, Costa Rica. 68 p.

Criollo, H. y García, J. (2029). Efecto de la densidad de siembra sobre el crecimiento de plantas de rábano (Raphanus sativus L.) bajo invernadero. Revista Colombiana de Ciencias Hortícolas. 3 (2):210217.

Eber, D., Giovanaz, M., Fachinello, J. y Nachtigal, J. (2016). Densidade de plantio e produção do maracujazeiro-azedo no Sul Do Brasil. Revista Brasileira de Fruticultura, 38(1), 99106. https://doi.org/10.1590/0100-2945-283/14

Fernández, G., Melgarejo, Luz., y Rodríguez, N. (2014). Algunos aspectos de la fotosíntesis y potenciales hídricos de la granadilla (Passiflora ligularis Juss.) en estado reproductivo en el Huila, Colombia. Revista Colombiana de Ciencias Hortícolas, 8(2), 206-216.

Fischer, G. y Alvarez, J. (2008). Efecto de la nutrición sobre la calidad de los frutos. pp. 92-104. En: Proc. XXXVIII Congreso Anual de Comalfi, 27-29 de agosto de 2008, Montería, Colombia.

Guerrero, R. (2001). Fertilidad de suelos, diagnóstico y control: fundamentos técnicos para la fertilización de cultivos. Guadalupe Bogotá - Colombia.

Holdridge, (1967). Ecología basada en las zonas de vida Centro Científico Tropical Costa Rica 216 pp.

Lim, T. (2012). Passiflora ligularis. En: Lim T. editors. Edible Medicinal And NonMedicinal Plants. Holanda, Springer. p. 174-177.

MINAGRI (Ministerio de Agricultura y Riego) (2018). Análisis del Mercado 2014 - 2018 Granadilla. Sierra y Seva Exportadora Unidad de Inteligencia Comercial. 34 pp.

Miranda, D. (2012). Granadilla (Passiflora ligularis Juss). En Manual para el cultivo de frutales en el trópico. Editorial Produmedios. pp. 550-578. 
Miranda, D. (2009). Manejo integral del cultivo de la granadilla (Passiflora ligularis Juss.). Cultivo, poscosecha y comercialización de las pasifloráceas en Colombia: maracuyá, granadilla, gulupa y curuba. Sociedad Colombiana de Ciencias Hortícolas, Bogota, 121-157.

Morillo, R. (2017) Efecto del manejo con dos y tres ejes en el rendimiento de granadilla (Passiflora ligularis Juss) en el Sagrario, Cantón Ibarra, Provincia de Imbabura. Tesis de Ingeniero Universidad Técnica del Norte Facultad de Ingeniería en Ciencias Agropecuarias y Ambientales. Ecuador $66 \mathrm{p}$.

Nunes, C., Cavalcante, F., Pereira, E., Souza, J., Almeida, J., de, Oresca, D. y Fernándes, P. (2017). Gas exchange and productivity of yellow passion fruit irrigated with saline water and fertilized with potassium and biofertilizer. Ciencia e investigación agraria, 44(2), 168-183.

Noda, Y. y Martín, J. (2014). Influencia de la densidad de plantación y la fertilización nitrogenada en el rendimiento de Morus alba var. tigreada. Pastos y Forrajes, 37(3), 291-297.

Ocampo, J., Coppens, d’E.G., Restrepo, M., Jarvis, A., Salazar, M. y Caetano, C. (2007). Diversity of Colombian Passifloraceae: biogeography and an updated list for conservation. Biota Colombiana 8(1): 1- 45. pp.

Ocampo, J., Arias, J., y Urrea, R. (2015). Colecta e identificación de genotipos élite de granadilla (Passiflora ligularis Juss.) en Colombia. Revista Colombiana de Ciencias Hortícolas, 9(1), 9-23.

Peña, R. y Galecio, M. (2019). Efecto del silicio orgánico en el rendimiento de maracuyá (Passiflora edulis), cultivada en Somate - Sullana. Revista de investigaciones de la Universidad Le Cordon Bleu, Perú. vol 6(1), 25 - 37.

Rajan. S., Tiwari, D., Singh, K., Saxena, P., Singh, S., Reddyl, Y., Upetril, K., Burondkar, M., Bhagwan A. y Kennedy, R. (2011). Application of extended BBCH scale for phenological studies in mango (Mangifera indica L.). Journal of Applied Horticulture. 13 (2): 108-114 2011

Rivera, B., Miranda, D., Ávila, A. y Nieto, M. (2002). Manejo integral del cultivo de la granadilla (Passiflora ligularis Juss). Primera Edición, Ed. Litoas, Manizales Colombia, 130 p.

Rivera, V. (2016). Implementación de un plan de manejo técnico para la producción sustentable de granadilla (Passiflora ligularis Juss) como alternativa de innovación frente a los sistemas tradicionales en el municipio de Caicedo-Antioquia. Yapal-Colombia 65 p.

Romero, E. (2019). Sostenibilidad de la agricultura familiar: el caso del cultivo de granadilla (Passiflora ligularis Juss) en la provincia de Oxapampa, Pasco, Perú. Tesis de Doctorado. Universidad Nacional Agraria La Molina - Perú 193 pp. 
Sancho, H. (2007). Curvas de absorción de nutrientes: Importancia y uso en los programas de fertilización. Informaciones agronómicas 36. IPNI. p. 6.

Sotomayor, M. (2019). Informe Estadístico- Proyecto Servicio de investigación para la adopción de un paquete tecnológico validado para la producción de granadilla orgánica como estrategia de diversificación productiva en la Comunidad Campesina San Miguel de Tabaconas. Consultor de Proyectos Multidisciplinarios de Investigación Científica - Universidad Nacional de Piura 10 pp.

Silva, L., Cavalcante, H., Lima, M., Barbosa, F., de Souza, C., dos Santos Sousa, E., y Cavalcante, F. (2016). Effect of humic substances and nitrogen fertilization on yellow passion fruit cultivation in the Brazilian semiarid región. African Journal of Agricultural Research, 11(35), 3307-3313.

Suárez. R. y Tomalá, G. (2012). Respuesta de Maracuyá INIAP- 2009 (Passiflora edulis f. flavicarpa Deg) a la Aplicación de NPK más Microelementos en el Primer año de Producción en San Vicente de Colonche. Universidad Estatal Península de Santa Elena Portada. Tesis de Ingeniero La Libertad - Ecuador 90 pp.

Suárez, C., Pérez, O. y Gómez, U. (2016). Sistemas de polinización en granadilla (Passiflora ligularis Juss.) como base para estudios genéticos y de conservación. Acta Agronómica, 65(2), 197-203.

Tello, G. (2013). Efecto de diferentes distanciamientos de siembra y altura de soporte sobre la producción y calidad de la maracuyá Pasiflora edulis f flavicarpa deg maracuyá mejorada INIAP 2009 Tesis de Ingeniero. Universidad Técnica de Manabí - Santa Ana - Manabí -Ecuador 63 pp.

Tirol, A., Ladha, J., Regmi, A., Bhandari, A., Inubushi, K. (2007). Organic Amendments Affect Soil Parameters in Two Long-Term Rice-Wheat Experiments. Soil Science Society of America Journal 71: 442-452 pp. 